\title{
Mortalidad post infarto del miocardio en Chile: Comparación de los registros de angioplastía primaria versus trombolisis
}

\author{
Alejandro Martínez $\mathbf{S}^{1}$, Carolina Nazzal N², Alejandro Fajuri $\mathbf{N}^{1}$, Luis Eduardo Barra $\mathrm{L}^{2}$, \\ Alejandro Mayerson $\mathrm{G}^{2}$, Gabriel Cavada ${ }^{3} \mathrm{Ch}$, Pabla Campos $\mathrm{T}^{4}$, Gastón Dussaillant $\mathrm{N}^{3}$, en \\ representación de los registros RENAC y GEMI. \\ ${ }^{1}$ Departamento de Enfermedades Cardiovasculares, Hospital Clinico Universidad Católica de \\ Chile. \\ ${ }^{2}$ Estudiantes de Medicina, Pontificia Universidad Católica de Chile. \\ ${ }^{3}$ Escuela de Salud Pública. Universidad de Chile \\ ${ }^{4}$ Departamento de Enfermedades cardiovasculares, Hospital J.J. Aguirre. Universidad de Chile.
}

Recibido el 4 de Enero de 2010, Aceptado el 9 de Marzo de 2010

Rev Chil Cardiol 2010; 29: 29-36

\section{Resumen}

Antecedentes: Desde el año 2005, se ha implementado en nuestro país un conjunto de políticas públicas para permitir un acceso universal al tratamiento trombolítico a los pacientes con infarto agudo del miocardio (IAM). Para evaluar sus resultados es importante establecer los estándares locales de las distintas opciones de reperfusión.

Objetivos: 1) Comparar la mortalidad precoz y alejada de los pacientes con IAM sometidos a angioplastía primaria (angioplastía) versus los sometidos a trombolisis, en Chile. 2) Establecer los factores de riesgo de mortalidad en estos pacientes.

Métodos: Utilizando los registros nacionales de angioplastía (RENAC) y de IAM (GEMI), seleccionamos a todos los pacientes con IAM tratados con angioplastía o trombolisis, durante los años 2003 y 2004. Entre ellos, comparamos mediante prueba de $t$ de Student o chi-cuadrado, según correspondiera, sus características basales y su mortalidad hospitalaria, a 30 días, y a 12 y 24 meses. Además se hizo análisis de regresión logística multivariado para identificar los factores que independientemente se asociaron a mayor riesgo de mortalidad.

Resultados: En el estudio se incluyeron 857 pacientes con IAM que recibieron trombolisis y 700 tratados con angioplastía. No hubo diferencias entre los grupos en cuanto a edad, sexo, incidencia de diabetes mellitus, hipertensión arterial, hiperlipidemia ni antecedentes de IAM previo. La mortalidad fue significativamente menor en Ios pacientes tratados con angioplastía (HR crudo: 0,65; 95\% IC: 0,49-0,86, $p=0,03$ ). La diferencia fue evidente tanto para la mortalidad precoz como para la alejada. Así, alcanzó un 10,6 vs 6,3\% ( $p<0,01)$, durante la hospitalización inicial, un 11,2 vs $8,1 \%$ a los 30 días ( $p<0,01)$, un 15,3 vs $10,6 \%(p<0,01)$ a los 12 meses, $y$ un 21,1 vs $11,7 \%$ a los 24 meses $(p<0,001)$, para trombolisis y angioplastía respectivamente. Otros factores que independientemente se asociaron a menor sobrevida fueron la mayor edad, el sexo femenino y la coexistencia de diabetes mellitus.

Conclusión: Entre los pacientes con IAM incluidos en los registros chilenos de angioplastía primaria y trombolisis, la mortalidad precoz y alejada fue significativamente menor en aquellos sometidos a angioplastía

Correspondencia: Dr. Alejandro Martínez S.

Departamento de Enfermedades Cardiovasculares,

Hospital Clínico Pontifica Universidad Católica de Chile

Correo Electrónico: amartine@med.puc.cl 


\section{Mortality in Myocardial Infarction in Chile: a comparison from the angioplasty and thrombolysis registries.}

Background: From 2005, thrombolysis has been made available as a public health policy to treat acute myocardial infarction (AMI) in Chile. A comparison with results obtained locally with primary PTCA is relevant

Aim: to compare early and late mortality in AMI between thrombolysis and PTCA treatments and to determine risk factors associated to mortality

Methods: Data from national registries of PTCA (RENAC) and trombolysis (GEMI) of patients treated from 2003 through 2004 were analyzed. Early (<30day) and late (12 and 24 months) mortality was compared between groups. Logistic regression analysis was used to identify independent risk factors for mortality.

Results: 857 patients received thrombolysis and 700 were treated by PTCA. Age, sex, prevalence of diabetes, hypertension, dyslipidemia and prior AMI were similar in both groups. PTCA was associated to lower mortality rates compared to thrombolysis (crude HR 0.65, 95\% C.I. 0.49-0.86, $p=0.03$ ). Early, 12month and 24month mortality rates for thrombolysis and PTCA treated patients were 10.6 vs. $6.3 \%, 15.3$ vs. $10.6 \%$ and 21.1 vs. $11.7 \%$, respectively $(p<0.01)$. Increased age, female gender and presence of diabetes were independently associated to mortality, overall.

Conclusion: Primary PTCA was associated to lower early and late mortality rates compared to thrombolysis in Chilean registry data.

Key words: Myocardial Infarction, angioplasty, thrombolysis

\section{Introducción}

Asociado a una mayor prevalencia de los factores de riesgo en la población chilena, en los últimos años se ha registrado un aumento sostenido de la mortalidad por cardiopatía isquémica, que en la actualidad representa la primera causa de muerte en nuestro país. De acuerdo a las estadísticas del Ministerio de Salud, sólo el año 2004 fallecieron 7.948 personas por esta causa. Una proporción importante de esta mortalidad está dada por el infarto agudo del miocardio con supradesnivel del segmento ST (IAM), que se estima alcanza una incidencia de 12.000 casos anuales y tiene una tasa de letalidad cercana al $50 \%$ dentro del primer año de su presentación, si se incluye la mortalidad prehospitalaria ${ }^{1}$.

Para enfrentar este problema, y considerando que la reperfusión precoz, sea con trombolisis o angioplastía primaria, es la medida más eficaz para mejorar su pronóstico ${ }^{2}$, desde el año 2005 se ha implementado en Chile una estrategia de reperfusión, contenida dentro del Régimen de Garantías Explícitas en Salud (GES), que consiste en el acceso universal de los pacientes con IAM al tratamiento con trombolisis endovenosa lo más pronto posible después de iniciados los síntomas. Para evaluar los resultados de esta estrategia es importante establecer los estándares locales de las distintas alternativas de reperfusión.

Así, el propósito de este estudio fue comparar la mortalidad precoz y alejada de los pacientes con IAM sometidos a angioplastía primaria versus los sometidos a trombolisis en hospitales chilenos. Al mismo tiempo, se 
pretendió identificar factores de riesgo independientes de mortalidad en estos pacientes.

\section{Material y Métodos}

Utilizando las bases de datos de los registros nacionales de angioplastía coronaria (RENAC) y de infarto (GEMI), realizadas por la Sociedad Chilena de Cardiología y Cirugía Cardiovascular, seleccionamos a todos los pacientes con IAM y SDST tratados en las primeras 24 horas de evolución con angioplastía primaria o trombolisis, durante los años 2003 y 2004. Entre ellos comparamos, mediante $t$ de Student para variables continuas y chi-cuadrado para variables categóricas, sus características basales y la mortalidad hospitalaria, a 30 días, y a 12 y 24 meses. La información sobre las características basales y mortalidad hospitalaria se obtuvo desde los datos consignados durante la hospitalización en cada registro y la mortalidad alejada mediante las bases del Departamento de Estadísticas del Ministerio de Salud. Para ambos grupos se construyeron las curvas de sobrevida con el método de Kaplan -Meier. Se ajustaron los resultados de acuerdo a las características basales de los pacientes, utilizando el modelo multivariado de regresión de Weibull. Los resultados se expresan como Hazard ratios (HR) y sus Intervalos de Confianza de $95 \%$.

\section{Resultados}

Pacientes: Se incluyeron 857 pacientes que recibieron trombolisis y otros 700 tratados con angioplastía. Aunque no se demostraron diferencias significativas en sus características clínicas y demográficas basales (tabla 1), ambos grupos diferían en relación al tipo de hospital de origen. La mayoría de los pacientes tratados con trombolisis provenía de hospitales públicos y sólo un $48 \%$ pertenecía a la región metropolitana (figura 1), mientras que la mayor parte de los sometidos a angioplastía tuvieron dicha intervención en hospitales privados, que en el $88 \%$ correspondía a la región metropolitana (figura 2).

Tratamiento: Todos los pacientes tratados con trombolisis recibieron Estreptokinasa endovenosa. Entre los sometidos a angioplastía se utilizaron stents en el $80 \%$ de los casos y el procedimiento fue exitoso (menos de $50 \%$ de estenosis residual con flujo final normal) en el $89 \%$ de los pacientes.

Tabla 1: Características demográficas y clínicas de los pacientes.

\begin{tabular}{lccc}
\hline & $\begin{array}{c}\text { Trombolisis } \\
\mathrm{n}=857\end{array}$ & $\begin{array}{c}\text { Angioplastía } \\
\mathrm{n}=700\end{array}$ & $\mathrm{p}$ \\
\hline Edad (años) & $60 \pm 11$ & $60 \pm 13$ & $\mathrm{~ns}$ \\
Sexo femenino (\%) & 23 & 25 & $\mathrm{~ns}$ \\
Diabetes Mellitus (\%) & 25 & 22 & $\mathrm{~ns}$ \\
Hipertensión (\%) & 49 & 51 & $\mathrm{~ns}$ \\
Hiperlipidemia (\%) & 27 & 40 & $\mathrm{~ns}$ \\
IAM previo (\%) & 3 & 4 & $\mathrm{~ns}$ \\
\hline
\end{tabular}


A. Martínez, C. Nazzal, A. Fajuri, L. Barra, A. Mayerson, G. Cavada, et. al.

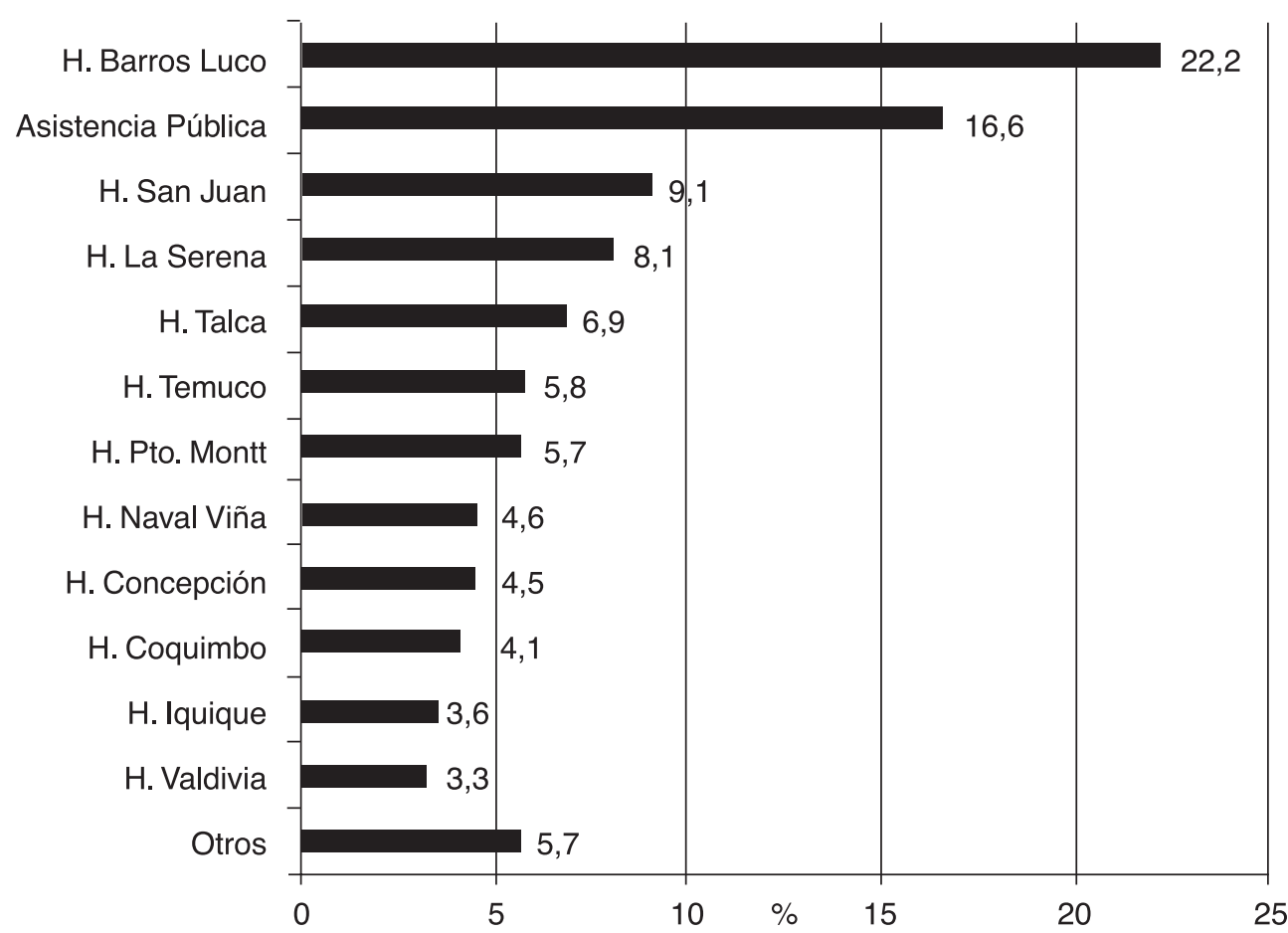

Figura1: Hospital de origen de los pacientes sometidos a trombolisis $(n=857)$

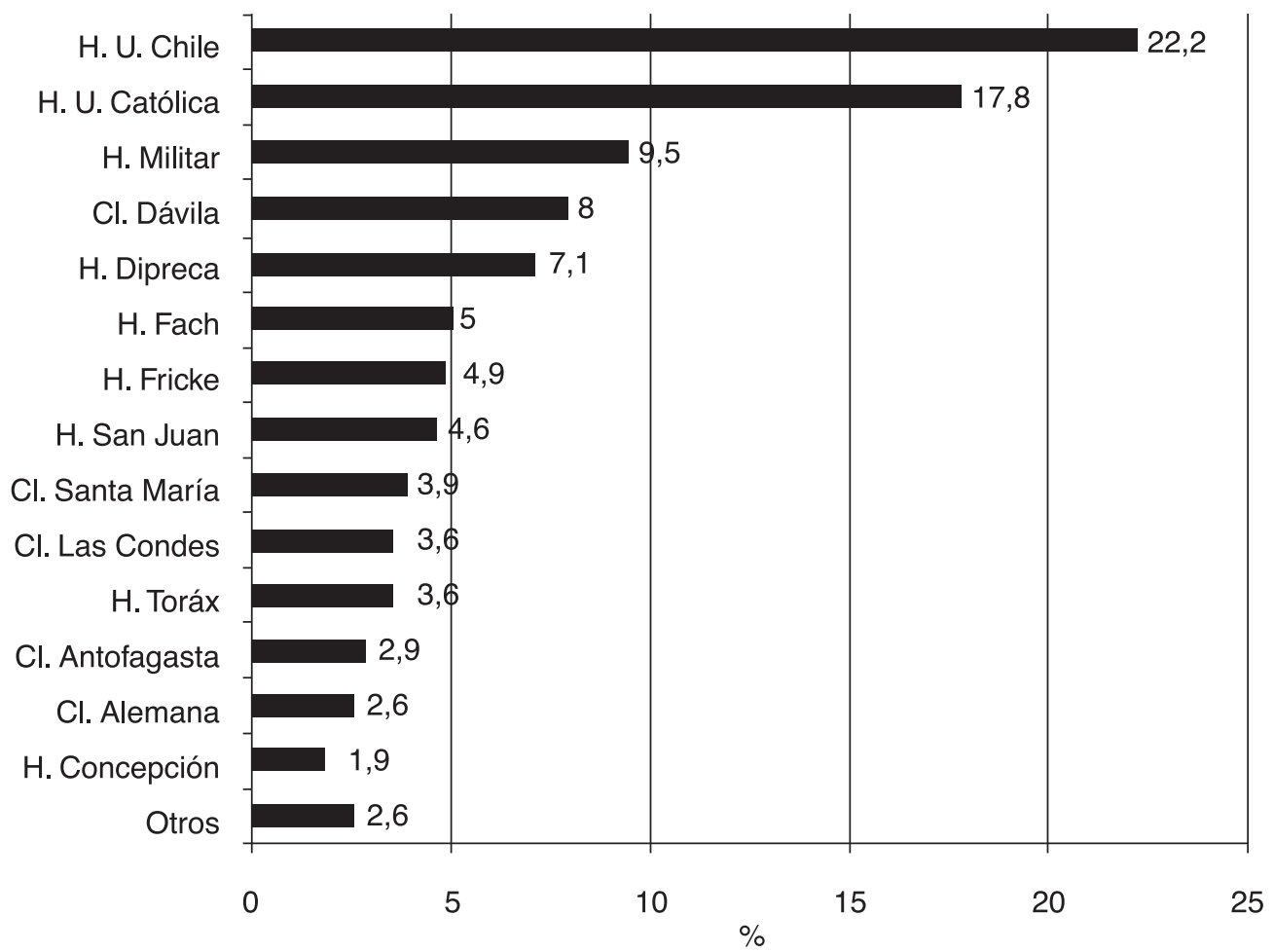

Figura 2: Hospital de origen de los pacientes sometidos a angioplastía $(n=700)$ 


\section{Mortalidad}

La mortalidad fue significativamente menor en los pacientes tratados con angioplastía comparados con los que recibieron trombolíticos, (HR crudo: 0,65; 95\% IC: 0,49-0,86, $p=0,03)$. La diferencia fue evidente tanto para la mortalidad precoz como para la alejada. Mortalidad en $\mathbf{3 0}$ días: la mortalidad dentro de los primeros 30 días del IAM alcanzó a un $11,2 \%$ en los pacientes tratados con trombolisis y a un $8,1 \%$ en los sometidos a angioplastía $(p<0,01)$. En ambos grupos la mayor parte de esta mortalidad se produjo durante la hospitalización inicial, alcanzando en este período un 10,6 y un $6,3 \%$, para la trombolisis y angioplastía respectivamente $(p<0,01)$.

Mortalidad alejada: la diferencia de mortalidad registrada a los 30 días, se acentuó a los 12 meses. En este período llegó a $15,3 \%$ en los tratados con trombolisis y sólo a $10,6 \%$ en los que tuvieron angioplastía $(p<0,01)$. La diferencia se incrementó aún más a los 24 meses de seguimiento, cuando alcanzó un $21,1 \%$ en los tratados con trombolisis y un $11,7 \%$ en los sometidos a angioplastía ( $p<0,01$. Este comportamiento se aprecia claramente en las curvas de sobrevida de Kaplan-Meier (figura 3), donde la diferencia a los 30 días se va acentuando progresi-vamente en el seguimiento alejado (Log Rank, $p=0,003)$.

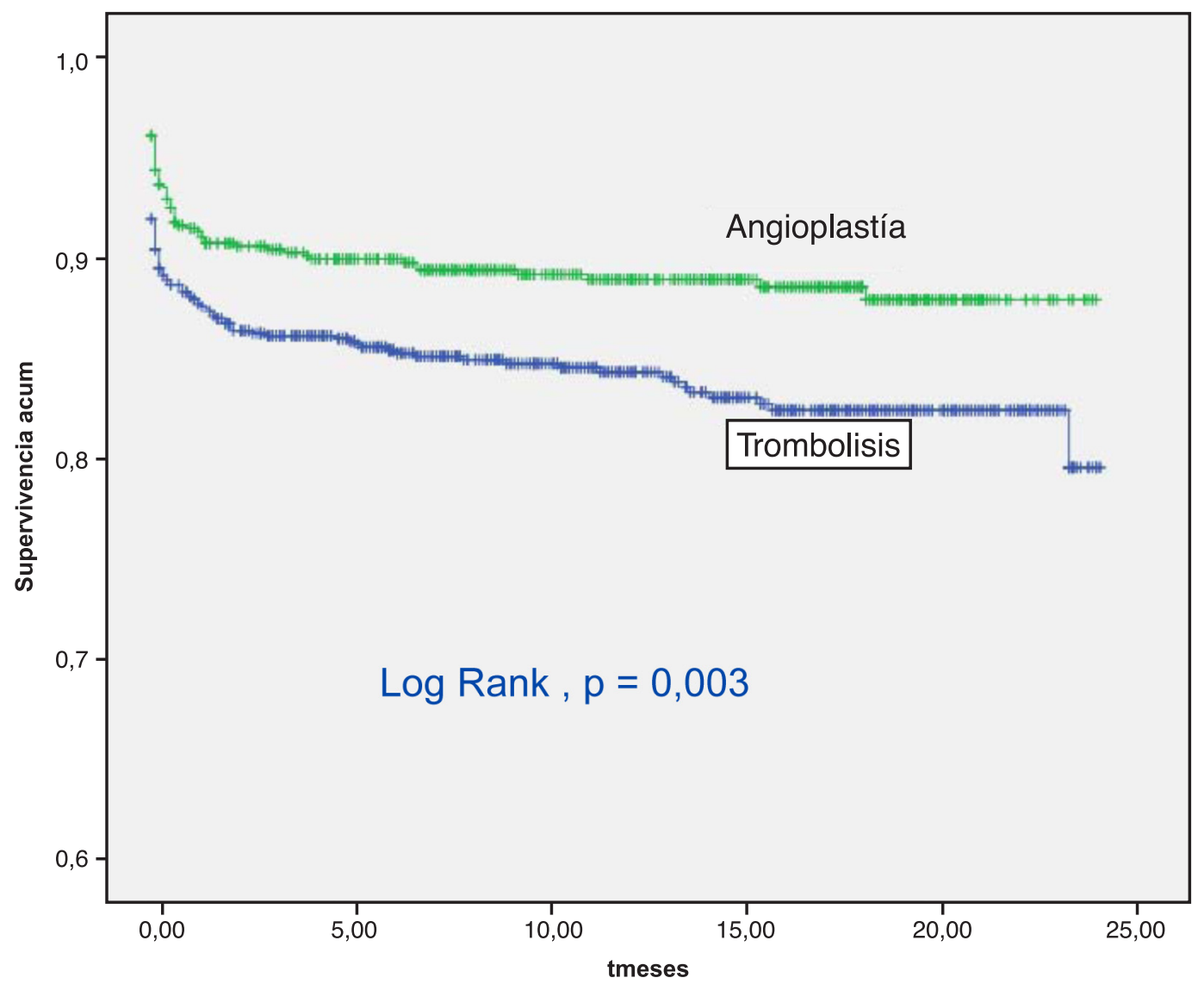

Figura 3: Curvas de Sobrevida, Kaplan-Meier, en pacientes sometidos a angioplastía y trombolisis. 
Factores de riesgo de mortalidad: en el análisis multivariado, ajustando por las características basales de los pacientes, persiste el efecto protector de la angioplastía sobre la trombolisis (HR: 0,58; 95\% IC:
$0,43-0,77, \quad p<0,001)$. Además resultan predictores independientes de mortalidad la edad avanzada, el sexo femenino y la presencia de diabetes mellitus (Tabla 2).

Tabla 2: Factores predictores de sobrevida. Análisis multivariado

\begin{tabular}{lccc}
\hline & Hazard Ratio & $95 \%$ IC & $p$ \\
\hline Angioplastía vs trombolisis & 0,58 & $(0,43-1,07)$ & $<0,001$ \\
Edad & 1.06 & $(1,04-1,07)$ & $<0,001$ \\
Diabetes Mellitus & 1,49 & $(1,12-2,29)$ & $<0,001$ \\
Sexo Femenino & 1,71 & $(1,28-2,29)$ & $<0,001$ \\
\hline
\end{tabular}

\section{Discusión}

El hallazgo más importante de este estudio es que entre los pacientes con IAM incluidos en los registros chilenos de angioplastía primaria y trombolisis, la mortalidad hospitalaria y alejada resultó significativamente menor en aquellos sometidos a angioplastía. El mismo hallazgo fue reportado en un meta-análisis de 23 estudios internacionales que compararon ambas intervenciones en forma randomizada ${ }^{3}$. Probablemente la mayor efectividad de la reperfusión y el menor riesgo de hemorragia asociado a la angioplastía, son los determinantes más importantes de estos resultados.

Frente a esta evidencia una primera reacción podría ser la de promover un esfuerzo mayor para implementar en nuestro país a la angioplastía primaria como método universal de reperfusión. Sin embargo, esta opción está limitada por su aplicabilidad. Para que la reperfusión sea beneficiosa es esencial que se haga en forma precoz ${ }^{4,5}$, lo que en muchos casos no es posible para la angioplastía. Las facilidades técnicas para este método, tal como ocurre en la mayoría de los países del mundo, están limitadas sólo a algunos hospitales, por lo que de promoverse en forma exclusiva esta estrategia, haría necesario trasladar a los pacientes, sin demora alguna, a esos centros. Basados en numerosos estudios, las guías de la AHA/ACC recomiendan la realización de angioplastía como el mejor método de reperfusión, siempre que pueda realizarse dentro de 90 minutos desde el momento de la primera consulta ${ }^{6}$, lo que desde un punto de vista logístico es muy difícil de implementar. Para controlar este inevitable retraso se ha evaluado el rol de la administración de trombolisis en el centro de la primera consulta, con posterior traslado para la realización de angioplastía (angioplastía facilitada), pero la efectividad de esta estrategia no se ha demostrado ${ }^{7}$.

Por lo anterior, parece razonable preguntarse si es posible mejorar los resultados que se han obtenido en Chile con la trombolisis. Comparando con igual tipo de tratamiento, la mortalidad de nuestros pacientes tratados con angioplastía es similar a la reportada en estudios internacionales, sin embargo, la obtenida en los sometidos a trombolisis es significativamente mayor. Así, en relación a los estudios randomizados citados $^{3}$, tanto a los 30 días como especialmente al año, la mortalidad chilena de los pacientes sometidos a trombolisis fue más alta $(15,3 \%$ versus $12 \%)$. La misma diferencia se aprecia cuando se comparan nuestros resultados con los encontrados en los pacientes tratados en el estudio GUSTO- $1^{8}$, en que se registró una mortalidad de sólo $2,9 \%$ entre los 30 días y un año, en 37.869 pacientes tratados con estreptokinasa o tPA.

Varios factores podrían explicar estas diferencias. Entre ellos, en este grupo puede ser muy importante 
la calidad del tratamiento farmacológico durante la hospitalización y después del alta. Se ha demostrado claramente el rol beneficioso del tratamiento médico con antiagregantes plaquetarios, estatinas, betabloqueadores e inhibidores de enzima convertidora, en la evolución alejada de los pacientes que han presentado un $\mathrm{IAM}^{6}$. En este estudio no evaluamos el tratamiento farmacológico, pero considerando que en esa época varios de estos fármacos no estaban disponibles en el sistema público, origen de la mayoría de los pacientes, debemos suponer que muchos de los casos trombolizados no los recibieron. Otro factor influyente pudiera ser una insuficiente pesquisa de isquemia residual sin realizar los debidos procedimientos de revascularización posterior a la trombolisis cuando proceden. En una serie nacional de pacientes trombolizados entre los años 2001 y 2002, encontramos que sólo el $23 \%$ tuvo algún procedimiento de revascularización durante el año posterior al evento ${ }^{9}$, lo que está por debajo de los reportes internacionales.

Estos dos factores pueden haberse modificado después del año 2005, con la implementación del GES, que da inicio al manejo protocolizado del IAM en nuestro país. Hoy existe completa disponibilidad de los fármacos y se ha mejorado el acceso a procedimientos de revascularización cuando son necesarios. Así, en una primera revisión que comparó la evolución intrahospitalaria de los pacientes con IAM, previo y posterior a la instauración del GES, se observó menor mortalidad, asociado a mayor uso de trombolíticos, fármacos asociados $y$ angioplastía de rescate $\mathrm{o}$ diferida ${ }^{10}$. Al mismo tiempo, es posible que el protocolo actual, que indica el uso de trombolíticos en el hospital de primera consulta, esté determinando una mayor precocidad en el tratamiento. Por lo tanto, varias razones hacen sospechar que en las series más modernas el pronóstico alejado de los pacientes sometidos a trombolisis puede haber mejorado.

Otra razón que dificulta la extensión de estos resultados a la situación actual, es que en este momento la angioplastía primaria no sólo está disponible en unos pocos centros seleccionados, como en la experiencia que presentamos, sino que se ha extendido ampliamente a otros hospitales privados y públicos, de cuyos resultados alejados desconocemos.

Este estudio además comprueba que los antecedentes de mayor riesgo en la evolución post infarto son la mayor edad, el sexo femenino y la presencia de diabetes mellitus, como está ampliamente demostrado ${ }^{11-13}$.

En conclusión, de acuerdo a esta comparación no randomizada, se reproduce en Chile el mejor resul-tado de la angioplastía primaria como método de reperfusión en el IAM. Sin embargo, creemos recomendable mantener la política de realizar esta intervención siempre que pueda efectuarse precozmente y mantener la trombolisis cuando la angioplastía no esté disponible. Será muy importante comparar estas cifras con el seguimiento alejado de los pacientes incluidos después del GES, antes de pensar en modificaciones del protocolo.

\section{REFERENCIAS:}

1.- Estadísticas del Ministerio de Salud. www.minsal.cl

2.- BOERSMA E, MERCADO N, POLDERMANS D, GARDIEN M, VOS J, SIMOONS ML. Acute myocardial infarction. Lancet 2003; 361: 847-58.

3.- KEELEY EC, BOURA JA, GRINES CL. Primary angioplasty versus intravenous thrombolytic therapy for acute myocardial infarction: a quantitative review of 23 randomised trials. Lancet 2003; 361: 13-20.

4.- BRODIE BR, HANSEN C, STUCKEY TD, RICHTER S, VERSTEEG DS, GUPTA N, et al. Door-to-balloon time with primary percutaneous coronary intervention for acute myocardial infarction impacts late cardiac mortality in highrisk patients and patients presenting early after the onset of symptoms. J Am Coll Cardiol 2006; 47: 289-95.

5.- Fibrinolytic Therapy Trialists' (FTT) Collaborative Group. Indications for fibrinolytic therapy in suspected acute myocardial infarction: collaborative overview of early mortality and major morbidity results from all randomised trials of more than 1000 patients. Lancet 1994; 343: 31122 
6.- ANTMAN EM, ANBE DT, ARMSTRONG PW, BATES ER, GREEN LA, HAND M, et al. ACC/AHA guidelines for the management of patients with ST-elevation myocardial infarction--executive summary: a report of the American College of Cardiology/American Heart Association Task Force on Practice Guidelines (Writing Committee to Revise the 1999 Guidelines for the Management of Patients With Acute Myocardial Infarction). Circulation 2004; 110: 588636.

7.- KEELEY EC, BOURA JA, GRINES CL. Comparison of primary and facilitated percutaneous coronary interventions for ST-elevation myocardial infarction: quantitative review of randomised trials. Lancet 2006; 367: 579-88.

8.- CALIFF RM, PIEPER KS, LEE KL, VAN DE WERF F, SIMES RJ, ARMSTRONG PW, et al. Prediction of 1-year survival after thrombolysis for acute myocardial infarction in the global utilization of streptokinase and TPA for occluded coronary arteries trial. Circulation 2000; 101: 2231-2238

9.- MARTÍNEZ A, GONZÁLEZ R, BARTOLUCCI J, DUSSAILLANT G, NAZZAL C. en representación de los Registros RENAC y GEMI. Eventos alejados post infarto del miocardio en Chile: registros de angioplastía primaria versus trombolisis. Rev Chil Cardiol 2005; 24: 374.
10.- CAMPOS P, NAZZAL C, SANHUEZA P, CORBALÁN R, BARTOLUCCI J, LANAS F, et al, en representación del Grupo GEMI. Impacto del plan AUGE en el tratamiento de los pacientes con infarto del miocardio con SDST en los hospitales chilenos. Rev Chil Cardiol 2007; 26: 254-255.

11.- MAGGIONI AP, MASERI A, FRESCO C, FRANZOSI MG, MAURI F, SANTORO E, et al. Age-related increase in mortality among patients with first myocardial infarctions treated with thrombolysis. The Investigators of the Gruppo Italiano per lo Studio della Sopravvivenza nell'Infarto Miocardico (GISSI-2). N Engl J Med 1993; 329: 14421448.

12.- WEAVER WD, WHITE HD, WILCOX R WEAVER WD, WHITE HD, WILCOX RG, AYLWARD PE, MORRIS D, GUERCI A, et al. Comparisons of characteristics and outcomes among women and men with acute myocardial infarction treated with thrombolytic therapy. GUSTO-I investigators. JAMA 1996; 275: 777-782.

13.- ZUANETTI G, LATINI R, MAGGIONI AP, SANTORO L, FRANZOSI MG. Influence of diabetes on mortality in acute myocardial infarction: data from the GISSI-2 study. J Am Coll Cardiol 1993; 22: 1788-1794. 